\title{
Dependence of critical current densities in Chevrel phase superconducting wires on magnetic fields up to $25 \mathrm{~T}$
}

\author{
A. Gupta ${ }^{a}, *$ N. Cheggour ${ }^{\mathrm{a}}$, M. Decroux ${ }^{\mathrm{a}}$, J.A.A.J. Perenboom ${ }^{\mathrm{b}}$, V. Bouquet ${ }^{\mathrm{c}}$, \\ P. Langlois ${ }^{d}, H$. Massat ${ }^{d}$, R. Flükiger ${ }^{a}, \emptyset$. Fischer ${ }^{a}$ \\ ${ }^{a} D P M C-G A P$, University of Geneva, 24 quai Ernest-Ansermet, 1211 Geneva-4, Switzerland \\ ${ }^{\mathrm{b}}$ High Field Magnet Laboratory, University of Nijmegen, Nijmegen, The Netherlands \\ ${ }^{'}$ Laboratoire de Chimie Minerale B, University of Rennes, France \\ ${ }^{\mathrm{d}}$ Lab. de Physicochimie des Materiaux, CNRS, 92195 Meudon Cedex, France
}

\begin{abstract}
$V-I$ characteristics have been measured, in several monofilamentry Chevrel phase superconductors (CPS) wires made using prereacted powders of both pure $\mathrm{SnMo}_{6} \mathrm{~S}_{8}$ (SMS) and $\mathrm{Pb}_{0.6} \mathrm{Sn}_{0.4} \mathrm{Mo}_{6} \mathrm{~S}_{8}$ (PSMS) compounds, in applied magnetic fields up to $25 \mathrm{~T}$. The values of $J_{\mathrm{c}}$ in different wires lie in the range $\sim 5 \times 10^{7}-10^{9} \mathrm{~A} / \mathrm{m}^{2}$ at $4.2 \mathrm{~K}$ and $9 \mathrm{~T}$. In the SMS wires the critical current density $J_{\mathrm{c}}(B)$ tends to vanish at the expected ideal $B_{\mathrm{c} 2}$. In contrast, for PSMS wires the $J_{\mathrm{c}}(B)$ tends to vanish at fields much smaller than the expected $B_{\mathrm{c} 2}$ for pure $\mathrm{PbMo}_{6} \mathrm{~S}_{8}$ (PMS) and nearer to that of the SMS. The $V-I$ characteristics at the dissipative onset are highly non-linear and show a power law behaviour $V=k I^{n}$ with a typical $n$-value $(1.8 \mathrm{~K}, 25 \mathrm{~T})>17$.
\end{abstract}

\section{Introduction}

It has been long realised that some of the CPS with high $B_{\mathrm{c} 2}(0)(35 \mathrm{~T}$ - SMS and 60T - PMS) fulfil the requirements for high field applications at $1.8-4.2 \mathrm{~K}$. In the optimised CPS wires we have now achieved record values of $J_{\mathfrak{c}}(4.2 \mathrm{~K}, 9 \mathrm{~T}) \geq 10^{9} \mathrm{~A} / \mathrm{m}^{2}$ [1]. However, the challenge for CPS lies in its use at field $>20 \mathrm{~T}$, where the $J_{\mathrm{c}}$ of $\mathrm{Nb}_{3} \mathrm{Sn}$ is intrinsically limited. In this work we address the three following questions: (1) In all reports on the best CPS wires until date $[2,3]$ the field $\left(B_{\mathrm{c} 2}{ }^{*}\right)$, at which $J_{\mathrm{c}} \Rightarrow 0$, was found to be much smaller than the ideal $B_{\mathrm{c} 2} ;(2)$ the scatter in the values of $J_{\mathrm{c}}$, especially when its $B$ dependence does not alter much, in differently processed wires; and (3) how sharp are the $V-I$ transitions at high fields in the CPS wires. Following the

* Corresponding author. line of reasoning developed in Refs. $[4,5]$ and the measured $V-I$ characteristics in the present work on pure SMS and several PSMS (Sn substituted (PMS) wires, we show that grain boundary superconducting properties and granularity (both macro and micro) lie at the origin of these questions.

\section{Experimental}

The powder and wire preparation was explained in detail in Ref. [6]. See Table 1 for the compositional and processing details of the wire reported in this work. Short wire samples $(3-13 \mathrm{~cm})$ were heat treated under hot isostatic pressing conditions at $900^{\circ} \mathrm{C}$ and $2 \mathrm{kbar}$ for $30-120 \mathrm{~min} . V-I$ characteristics were measured at 1.8 and $4.2 \mathrm{~K}$ with magnetic field $(B=1-25 \mathrm{~T})$ applied perpendicular to the current direction. The $J_{\mathrm{c}}$ is defined at a $10 \mu \mathrm{V} / \mathrm{cm}$ criterion. 
Table 1

Powder and composition of the wires

\begin{tabular}{lll}
\hline No. & CPS powder & Barriers \\
\hline 1 & SMS & $(\mathrm{Sw}) / \mathrm{Nb} / \mathrm{Cu} / \mathrm{Steel}$ \\
2 & PSMS $+0.2 \mathrm{Sn}$ & $(\mathrm{Ex}) / \mathrm{Nb} / \mathrm{Cu} / \mathrm{Steel}$ \\
3 & PSMS $+10 \% \mathrm{P}$ & $(\mathrm{Sw}) / \mathrm{Nb} / \mathrm{CuNi30}$ \\
4 & $\mathrm{PSMS}+0.2 \mathrm{Sn}+10 \% \mathrm{P}$ & $(\mathrm{Sw}) / \mathrm{Nb}^{*} / \mathrm{CuNi30}$ \\
5 & PSMS $+0.2 \mathrm{Sn}+10 \% \mathrm{P}$ & $(\mathrm{Sw}) / \mathrm{Nb}^{*} / \mathrm{CuNi30} / \mathrm{Steel}$ \\
6 & PSMS $+0.2 \mathrm{Sn}$ & $(\mathrm{Sw}) / \mathrm{Nb}^{*} / \mathrm{CuNiBe}$ \\
\hline
\end{tabular}

$\mathrm{P}-1.1 \mathrm{~Pb}+\mathrm{MoS}_{2} / \mathrm{Mo}$ precursors; $\mathrm{Nb}$ - Powder processed; $\mathrm{Nb}^{*}$ - melt processed; Ex - Extruded; $\mathrm{Sw}$ - Swaged.

\section{Results and discussion}

In Fig. 1(a) we show $J_{\mathrm{c}}$ as a function of $B$ at 4.2 and $1.8 \mathrm{~K}$ for both SMS and PSMS wires. For both the compounds, we observe nearly the same $J_{\mathrm{c}}(B)$ dependence. Only at high fields $>20 \mathrm{~T}$ the $J_{\mathrm{c}}(B)$ for SMS drops slightly faster as compared to the PSMS. Changing the voltage criterion for $J_{\mathrm{c}}$ definition do not change the results appreciably. In Refs. $[5,7]$ we had shown that the limiting mechanism for pinning, in polycrystalline CPS samples heat treated at temperatures $\leq 1000^{\circ} \mathrm{C}$ for reasonable times, could be a flux line shear along the grain boundaries. Thus we might use Kramer plots for determining $B_{\mathrm{c} 2}{ }^{*}$, which are shown in Fig. 1(b). For SMS wire $B_{\mathrm{c} 2}{ }^{*}=27.5 \mathrm{~T}(4.2 \mathrm{~K})$ and $=33 \mathrm{~T}(1.8 \mathrm{~K})$ approach the ideal $B_{\mathrm{c} 2}(0)=35 \mathrm{~T}$ in excellent agreement. However, for the PSMS wire $B_{\mathrm{c} 2}{ }^{*}=31.5 \mathrm{~T}(4.2 \mathrm{~K})$ and $=38 \mathrm{~T}(1.8 \mathrm{~K})$ are much smaller than the expected ideal $B_{\mathrm{c} 2}(0)=60 \mathrm{~T}$ for pure PMS and nearer to that of the SMS. This may indicate that the flux line shear channels in PSMS wire have a composition near to SMS, which can be caused by an excess of $\mathrm{Sn}$ at the grain boundaries. Note that our results of $B_{\mathrm{c} 2} *$ in SMS wire suggest that at temperatures of $1.8-4.2 \mathrm{~K}$, the irreversibility line $[8,7]$ for this compound lies very near to the expected ideal $B_{\mathrm{c} 2}(T)$ line.

In Fig. 2 we show $J_{\mathrm{c}}$ as a function of $B$ for different PSMS wires (see Table 1), all hot isostatically pressed at $900^{\circ} \mathrm{C}$ and $2 \mathrm{kbar}$ for $2 \mathrm{~h}$. In spite of the fact that the absolute values of $J_{\mathrm{c}}$ lie in a scatter window of more than one order of magnitude, the $J_{\mathrm{c}}(B)$ dependence is the same for all the wires. This clearly indicates that the main difference in these wires is that of the effective supercurrent - carrying cross-section. Several reasons depending on the magnitude of the $J_{c}$ scatter, like macrogranularity - cracks, porosity, and microgranularity - Jospehson coupled grain boundaries due to incomplete sintering or severe degradation, might be responsible.

In Fig. 3 are shown on a double logarithmic scale the $V-I$ characteristics for PSMS wire no. 2, which has

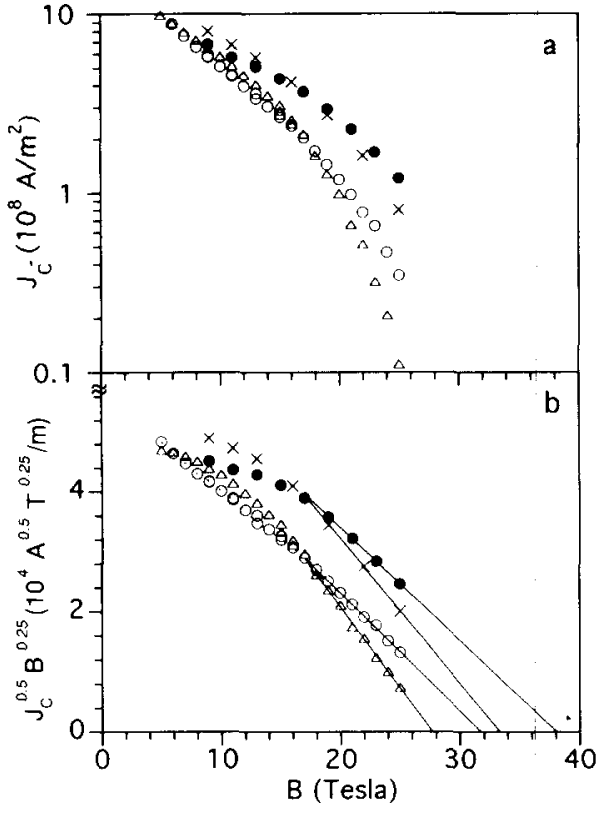

Fig. 1. (a) $J_{\mathrm{c}}$ versus $B$ for wire no. $1(\triangle)$ and $5(O)$ at $4.2 \mathrm{~K} ; 1(x)$ and $5(0)$ at $1.8 \mathrm{~K}$. (b) Kramer plots for the same data.

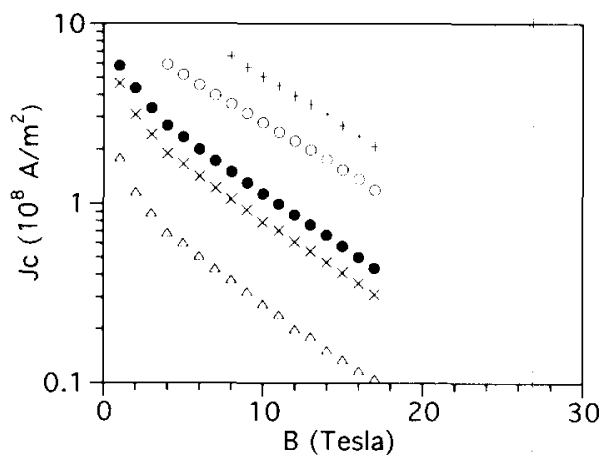

Fig. 2. $J_{\mathrm{c}}$ versus $B$ for wire no. $2(O), 3(\triangle), 4(\times), 5(+)$ and 6 (๑).

a $J_{\mathrm{c}}=3.5 \times 10^{8} \mathrm{~A} / \mathrm{m}^{2}$ at $4.2 \mathrm{~K}$ and $9 \mathrm{~T}$. The jumps in the $V-I$ observed at currents $>J_{\mathrm{c}}$ (see $V-I$ at $13 \mathrm{~T}$ and $14 \mathrm{~T}$ in Fig. 3) may be accounted by a "current induced flux flow avalanche" in the wires (see also Ref. [9]). The dissipative onset is rather abrupt and in the $10^{-5}-10^{-7} \mathrm{~V}$ window, the $V-I$ follow a power law $\left(V=k I^{n}\right)$. As shown in Fig. 4 the $n$-values decrease monotonically with $B$. Note also that the $n$-values at $1.8 \mathrm{~K}$ are a factor $\sim 3$ higher than at $4.2 \mathrm{~K}$. Similar $V-I$ behaviour also observed in single crystal high- $T_{\mathrm{c}}$ cuprates has been discussed within vortex glass and collective pinning theories, where the activation barrier for flux motion is shown to have a non-linear 


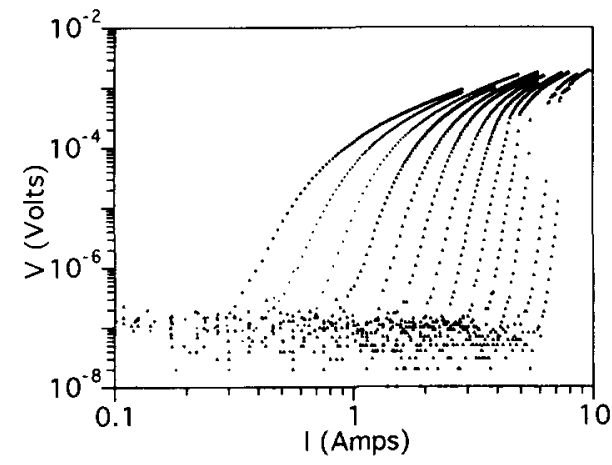

Fig. 3. $V-I$ characteristics for the wire no. 2 for $B=13 \mathrm{~T}$ (right) to $25 \mathrm{~T}$ (left) in steps of $1 \mathrm{~T}$.

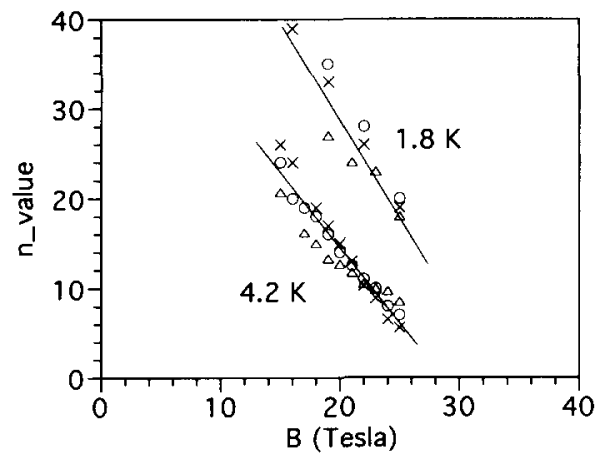

Fig. 4. $n$-values versus $B$ for wire no. $1(x), 2(O)$ and $5(\triangle)$. The solid lines are only a guide. dependence on $J / J_{\mathrm{c}}$. However, in our polycrystalline CPS samples, where the flux lines might flow along the grain boundaries, the distribution of $T_{\mathrm{c}}$ and $B_{\mathrm{c}}$ in the grain boundaries may be the origin of the observed behaviour [10]. These aspects need to be further explored.

\section{Conclusions}

The conclusions of this work are as follows:

1. In a typical $J_{\mathrm{c}}-B$ plot (see Fig. 1(a) of CPS wires), the $J_{\mathrm{c}}(B)$ curve will stretch vertically by a reduction in granularity and horizontally by improving the superconducting properties of the grain boundaries.

2. The relatively high $n$-values ( $\geq 6$ at $4.2 \mathrm{~K}$ and $\geq 17$ at $1.8 \mathrm{~K}$ ) in both SMS and PSMS wires at $25 \mathrm{~T}$ indicate the potential for their high field applications.

\section{References}

[1] N. Cheggour et al., to be published.

[2] G. Rimikis et al., IEEE Trans. Appl. Supercond. 27 (1991) 1116.

[3] H. Yamasaki et al., J. Appl. Phys. 70 (1991) 1606.

[4] M. Decroux et al., IEEE Trans. Appl. Supercond. 3 (1993) 1502.

[5] A. Gupta et al., Proc. ICMAS'93 (Paris) Pub. IITT Int. (1993) 402.

[6] N. Cheggour et al., Proc. ICMAS'93 (Paris) Pub. IITT Int. (1993) 402.

[7] A. Gupta et al., Presented at $\mathbf{M}^{2} \mathrm{HTSC}$ (Grenoble '94), Physica $C$, in press.

[8] C. Rossel et al., Physica C 165 (1990) 233.

[9] P.F. Hermann et al., Physica C 202 (1992) 61.

[10] R. Wördenweber, Cryogenics 32 (1992) 1098. 